\title{
Subtotal Colectomy and lleostomy in Early Pregnancy and Review of Relevant Medical Literature
}

\author{
Anthony Emeka Madu
}

Specialist Registrar in Obstetrics and Gynaecology, Scarborough and North East Yorkshire Healthcare NHS Trust, Yorkshire, United Kingdom

*Corresponding author: Anthony Emeka Madu, Specialist Registrar in Obstetrics and Gynaecology, Scarborough and North East Yorkshire Healthcare NHS Trust, Yorkshire, United Kingdom, Tel: +447931626315; E-mail: emymadu@yahoo.co.uk

Received date: May 13, 2016; Accepted date: Jun 21, 2016; Published date: Jun 23, 2016

Citation: Emeka Madu A. Subtotal colectomy and ileostomy in early pregnancy and review of relevant medical literature, Gynecol Obstet Case Rep. 2016, 2:2.

\section{Abstract}

Inflammatory bowel diseases, mainly ulcerative colitis and Crohn's disease occasionally require surgical intervention in the treatment of the patient. When surgery is necessary during pregnancy, there is understandably much concerns and anxieties shown by the carers and the patient regarding adverse effects of surgery on the pregnancy and the mother. I present a case of a subtotal colectomy and ileostomy performed at about 6 weeks gestation. I shall also review available literature on the impact of surgery and safety in continuing pregnancy following surgical intervention during pregnancy.

Keywords: Severe ulcerative colitis; Early pregnancy; Subtotal colectomy; lleostomy

\section{Introduction}

This is a case of early pregnancy complicated by exacerbated severe ulcerative colitis and subtotal colectomy and ileostomy. The incidence of inflammatory bowel diseases (IBD) has increased over the last four decades and is mainly due to increases in the incidence of ulcerative colitis and Crohn's disease. Though ulcerative colitis has much in common with Crohn's disease, it differs in that ulcerative colitis affects mainly the colon and rectum, leaving the rest of the gastrointestinal tract unaffected. On the other hand, Crohn's disease can affect the whole gastrointestinal tract from the mouth to the anus.

Surgical removal of the colon and rectum can cures ulcerative colitis. This means that the disease does not usually recur after surgery. However, Crohn's disease can recur after restorative surgical operation to remove diseased part of the bowel and connect the healthy ends. Ulcerative colitis occurs as an intermittent disease. There are therefore periods of remissions and exacerbations. Though ulcerative colitis can sometimes go into remission on its own, it often needs treatment before it goes into remission. Both lesions usually affect young adults. Ulcerative colitis generally affects more women than men while Crohn's disease affects both sexes equally. Caucasians are more affected than Afro-Caribbeans. It usually presents with abdominal pain, rectal bleeding and diarrhoea and weight loss.

Ulcerative colitis has an incidence of 1-20 cases per 100,000 individuals per year, and a prevalence of 8-246 per 100,000 individuals [1]. Like Crohn's disease, ulcerative colitis is classed as an autoimmune disease and managed as such. Both are treated with anti-inflammatory drugs, immunosuppression therapy, and with biological therapy targeting specific components of the immune response.

\section{Case History}

A 25 year old woman presented for antenatal booking at 15 weeks gestation around March. She had a past history of ectopic pregnancy treated by salpingectomy.

She had history of ulcerative (intermediate) colitis diagnosed two years earlier by diagnostic colonoscopy and biopsies of the left hemicolon and rectum. She had been in remission until a year prior to the index presentation. She said she had a long period of stress and difficulties with her partner and from her beloved father dying from a terminal bowel cancer, the type of which she could not disclose.

Last December, she had been admitted from the clinic in the morning by the medical team due to exacerbation of her ulcerative colitis in pregnancy at 4 weeks gestation. She had presented with abdominal pain of 8 weeks duration, rectal bleeding and diarrhoea of 4 weeks duration. Diarrhoea frequency was on average, 10-20 times per day. Her observations were body mass index (BMI) 26; pulse 108; basal glucose measurement 6.9; SpaO2 98\%; blood pressure (BP) $134 / 64$ and respiratory rate (RR), 18, at presentation. By $5 \mathrm{pm}$ the same day, her tachycardia had settled.

She had a history of generalised pruritus possibly due to scabies, possibly erythema, which was investigated by dermatologist and rheumatologist and treated. She had family history of arthritis (maternal aunt) possibly chronic juvenile arthritis.

She was allergic to penicillin, which gave her skin rash. 
She had a strong family history of bowel disorder, her sister and mother both suffered from ulcerative colitis. Her father died from suspected terminal bowel cancer.

She had two courses of steroids from her General Practitioner in addition to mesalazine $1 \mathrm{~g}$ three times daily and hydrocortisone twice daily.

Clinical assessment at index presentation by the medical team confirmed severe exacerbation of ulcerative colitis in pregnancy. Stool analysis revealed her diarrhoea was likely caused by clostridium difficile and this was added to the initial diagnosis. She had multidisciplinary care involving Medical, Surgical, Nutritional (Dietetics) and Gynaecological teams. Ultrasound performed next day following admission revealed a $5 \mathrm{~mm}$ gestational sac without yolk sac or fetal pole.

Flexible sigmoidoscopy revealed of severe active colitis. Pelvis-shielded abdominal $x$-ray did not reveal any other significant abdominal finding. Haemoglobin $(\mathrm{Hb})$ on the second day following admission was $7.5 \mathrm{~g} / \mathrm{dl}$ but liver function test and urea and electrolyte were normal. Platelet count was 444 and white cell count (wcc) was normal.

Despite aggressive and intensive conservative management, her colitis did not settle but deteriorated. $\mathrm{Hb}$ dropped slightly to $7.4 \mathrm{~g} / \mathrm{dl}$; wcc rose to 13.7 ; platelet count rose to 517 and creactive protein (crp), was 19 . She was very unwell. Blood transfusion was considered but was withheld until the involvement of the haematologist.

Abdominal ultrasound scan showed diffuse thickening of the transverse colon consistent with colitis but no evidence of toxic megacolon.

The following morning she became very unwell and she had bled per rectum 5 times that morning. $\mathrm{Hb}$ was $8.2 \mathrm{~g} / \mathrm{dl}$; platelet, 661; wcc 14.10, potassium 2.9

After a further drop in $\mathrm{Hb}$ to $7.2 \mathrm{~g} / \mathrm{dl}$, she was subsequently transfused 2 units of blood after discussion with the obstetric and gynaecological team. Post transfusion $\mathrm{Hb}$ was $9.4 \mathrm{~g} / \mathrm{dl}$.

9 days following admission, she complained of vaginal bleeding on wiping. Ultrasound scan showed a viable intrauterine pregnancy with a crown rump of $2 \mathrm{~mm}$, equivalent to 5-6 weeks gestation with provisional expected dated of delivery in August same year. She felt much better clinically. She was fasted and had counselling and discussion on possibility of miscarriage following the major surgery she was about to have. There were considerable discussions with her on the survival chances of the current pregnancy and the risks of miscarriage at that stage of pregnancy. She was counselled and informed that the emphasis was on her survival which was more important. Though she was understandably very keen to keep the pregnancy, she understood that her life and survival takes precedence.

At this stage, 10 days following admission and following resuscitation and stabilization, a decision was made to go ahead for more definitive surgical treatment of her severe ulcerative colitis. The decision was based on the rectal bleeding and stool frequency of 10-20 per day on admission; severe active colitis on flexible sigmoidoscopy and ultrasound evidence of diffuse thickening of transverse colon consistent with active colitis.

A subtotal colectomy and ileostomy was performed following a multidisciplinary discussions and plan. Her postoperative period was complicated my recurrent episodes of bowel obstruction. Ultrasound scan did not identify a mechanical cause for this. Eventually her bowel obstruction resolved following conservative management.

She was fit enough to be discharged after more than one week in hospital.

On review three weeks later, she was much better and had no further episode of abdominal pain. Her dose of steroids was reduced. She was reviewed again in a week in the joint Gastroenterology Clinic where further care with other specialists in the multidisciplinary care team was discussed. There was consideration to put her on mesalamine (Asacol) on her next follow up visit.

At next review, she had started eating and drinking normally. She complained that her ileostomy was large and asked for revision or reversal. She was then reassured and considered for ileoanal pouch following delivery of her baby as she was keen to keep her pregnancy.

At the antenatal care booking at about 12 weeks gestation, she declined Triple test (Triple Screen Test, USA). Routine TORCHS screen (toxoplasmosis, rubella, cytomegalovirus, herpes simplex, and HIV), was normal. Her dating scan results were normal and her expected date of delivery was in August, same year.

From obstetric point of view, maternal and fetal observations were essentially normal at booking. She was still under multi-disciplinary care.

From surgical point of view, her post-operative recovery following discharge from the hospital had been relatively uneventful but she still needed to undergo routine follow-up assessments by the surgical and other teams. She was very happy with fetal assessment and contented with her progress and recovery.

When reviewed at 15 weeks gestation, mother and fetus were doing well from obstetric point of view. She was reassured that at 15 weeks the rate of miscarriage was very low. From surgical point of view, she had continued to improve. She was booked for anomaly ultrasound scan at 20 weeks gestation. She had growth scans booked for gestations 24, 28 and 32 and 36weeks gestation to rule our fetal growth restriction. It was very difficult for the author to follow her progress for the rest of the pregnancy after the author left to work in another hospital and it appears she left the area and booked at a tertiary hospital with better facilities. The author had been unsuccessfully in his efforts to know the following later events:

(a) Chronology of further antenatal and surgical care

(b) Chronology of labour and delivery events 
(c) Chronology of postnatal events

(d) Outcome of her delivery and follow-up

(e) Other care from other teams, etc.

Enquiries of her whereabouts did not yield any positive results and the author would therefore not want to speculate on (a)-(e) above.

\section{Discussion}

This case is unique in the way it presented; the strong family history of bowel disorders; the resilience of the patient to carry on with the pregnancy despite her debilitating condition and the life changing surgery she had. It is very understandable why the patient remained resilient to carry on with the pregnancy; she had lost a pregnancy through an ectopic pregnancy which was treated by salpingectomy. So her fertility is already compromised by the ectopic pregnancy and its surgical treatment. The patient thought this could well be her last chance to be a mother.

The author concedes that the following events would have shed more light on what happened to her in her pregnancy:

(a) Chronology of antenatal and surgical care.

(b) Chronology of labour and delivery events, especially whether she was delivered by emergency or elective caesarean section or did vaginal delivery.

(c) Chronology of postnatal events, any further episode bowel obstruction or exacerbation in the puerperium ?

(d) Outcome of her delivery and follow-up, including the newborn and early neonatal care.

(e) At what stage following pregnancy did she have the ileoanal pouch?

(f) Any other care she received from other teams.

However, the author felt that the information available is significant to be shared with the scientific community.

IBD affects pregnancy by causing infertility, miscarriage fetal anomaly, preterm labour, stillbirth and low birth weight. Rates of miscarriage are not affected significantly in well controlled or non-active disease. Women who had surgery and nonactive disease in pregnancy tend to tolerate pregnancy well but are still at risk of bowel obstruction from the second trimester [2].

Markers of adverse maternal and fetal outcomes in pregnancy and puerperium are as follows; first presentation in pregnancy, which is not applicable to this case; active disease at conception, as in our patient; colonic rather than small bowel disease, as in this case; active disease after resection, which is not applicable to this case and severe disease treated by surgery, as in this case.

On the other hand, pregnancy may cause a relapse of IBD which can happen at any time during the pregnancy. These relapses are more common in the first trimester, as occurred in this case, and in the puerperium. The risk of exacerbation is not increased but similar to that of non-pregnant patients.

From historical perspective, cases of pregnancy following colectomy had long been cited in medical literature [3-5]. Fears regarding maternal and fetal well-being following major surgical intervention in severe inflammatory bowel disease had long existed. The author could only go as far back as 1955 [3].

Koganei $\mathrm{K}$ et al, [6] reported a case series of 45 patients aged 20-39 years who had total colectomy with ileal pouch or anal canal anastomosis for severe ulcerative colitis with various minor maternal and fetal complications including short term faecal soiling in the majority; preterm labour(1 patients) and caesarean section (4 patients). There were no pouchrelated complications. The types of delivery were caesarean section in four patients and vaginal delivery in one patient. There was one preterm delivery at 28 weeks gestation, but others were term deliveries. No abnormality was found in the babies. The authors concluded that surgical treatment of IBD in pregnancy was safe.

More recently, Dozois E et al, [7] reported a case series of 37 patients who were surgically managed in pregnancy. It concluded that maternal and fetal mortality rates were very low; that maternal morbidity was low and that subtotal colectomy and Brooke ileostomy for ulcerative colitis during pregnancy was safe. It went on to highlight the benefits of multidisciplinary approach to maximise maternal and fetal outcomes. A multidisciplinary team that includes a gastroenterologist, high-risk obstetrician and experienced surgeon were essential for optimal outcome, it concluded.

Greenfield C [8] reported a case of ulcerative colitis which was complicated by caesarean section delivery at 32 weeks gestation and 10 days postpartum, by surgical intervention by way of colectomy following puerperal exacerbation of ulcerative colitis. This surgical intervention was subsequently complicated by transient postoperative ileus. This confirms that exacerbation of her ulcerative colitis can occur in the puerperium, necessitating surgical intervention.

Boulton $\mathrm{R}$ et al, [9] reported a case of fulminant ulcerative colitis. The patient underwent had subtotal colectomy at 26 weeks gestation and subsequently had intra-abdominal sepsis following the surgery. However, she delivered prematurely at 32 weeks weeks gestation.

These citations variously illustrate the degree of fetal and maternal morbidity which can influence eventual outcomes.

Writing in the European Crohn's and Colitis Organisation; Inflammatory Bowel Disease in 2013, Wilson and colleagues [10] stated that it had been historically assumed that surgery for ulcerative carried much risk to mother and fetus. However, their review demonstrated that this was not the case currently and that surgical operation for ulcerative colitis can be performed during pregnancy without much maternal and fetal morbidity. It concluded that after surgery, it was safe for the pregnancy to continue. It advised that when clear indications exist, surgical intervention for ulcerative colitis must not be 
delayed because of fears of adverse effects on the pregnancy. They recommended subtotal colectomy and ileostomy. It was clear our surgical team acted in a similar way and performed similar operation. Our case, though seen three or four years earlier before this publication, is a clear demonstration of this fact, the up-to-date and good practice of our clinical teams demonstrated. Our surgical team had intervened around 6weeks gestation. At 15 weeks gestation, the fetus was growing well. Maternal and fetal progress was very good.

More recently, Motoi Uchino and his colleagues [11] reported two cases of apparent refractory ulcerative colitis they managed. They concluded that regardless of pregnancy, the need for emergency surgical intervention should be determined promptly based on the severity of colitis and the general maternal condition. Surgical operation and the severity of the ulcerative colitis should never be the determining factors for discontinuing pregnancy. The pregnancy can be continued for as long as possible, without significant fetal and maternal problems, during and after surgical operation for severe ulcerative colitis, it concluded.

The author's opinion is that the studies cited in this paper appeared to take no account of what may be going on in developing countries where standards of care are lower; community nutrition is poor and access to standard or even substandard care is a problem. In these poor countries, the historic fears of the effect of surgery on mother and baby may well remain relevant for some time to come.

Comparatively, mortality rates have evolved and improved. The recent overall mortality rate associated with surgical treatment of ulcerative colitis is less than $1 \%$ and the overall morbidity is about 30\% Fazio et al, [12]. Major complications include strictures, pelvic sepsis, pouch failure, fecal incontinence, pouch dysplasia or cancer development, sexual dysfunction and female infertility.

Patients who have had surgical procedures for IBD for instance; ileal pouch-anal anastomosis (IPAA) should not be discouraged from childbearing because of the pouch. Pregnancy and delivery are safe in patients with surgical procedures for IBD as stated earlier. Patients may experience a transient increase in stool frequency or incontinence during pregnancy, both of which resolve after delivery [13].

It remains quite controversial whether vaginal or caesarean section delivery is better for pregnant women with a pelvic pouch. Vaginal delivery has the potential risk of disrupting the anal sphincter, although the mode of delivery should be dictated primarily by obstetric considerations [14,15]. In my patient, on the balance, I would have expected that all efforts should be made to encourage vaginal delivery, a planned vaginal delivery, if no adverse obstetric factors are present, with recourse to caesarean section if vaginal delivery cannot be achieved. This is because caesarean section is froth with more risks of complications relating to the previous bowel surgery and caesarean section itself.

For instance, caesarean sections can worsen the major complications of previous major bowel surgical operation for IBD like; anal stricture (11\% following bowel surgery); adhesions; pelvic sepsis (9.5\% following bowel surgery); pouch failure (6.8-9.5\% following bowel surgery); faecal incontinence; pouch dysplasia; sexual dysfunction; and female infertility [16]. It can also lead to new complication like bowel or viscus perforation. It may be difficult to divide pelvic adhesions caused by bowel surgery at caesarean section.

I would expect any caesarean section to be performed by a multi-disciplinary team comprising of an experienced obstetrician, with experienced bowel surgeon, anaesthetist in attendance.

Aside from pregnancy and delivery; surgical operation for IBD for instance; IPAA has been associated with a small risk of sexual dysfunction. The risk is greatest in patients who require preoperative pelvic surgical operation. Postoperative impotence and retrograde ejaculation have been observed in approximately $1.5 \%$ and $4 \%$ of men respectively. Transient dyspareunia occurs in about $7 \%$ of women, although coital frequency and the ability to experience orgasm remain unchanged $[17,18]$. In women, fertility is significantly decreased after open bowel surgical operation for IBD, 19 although successful pregnancies do occur regularly [20].

\section{Conclusion}

Exacerbation of uncreative colitis in early pregnancy and subsequent subtotal colectomy and ileostomy is a major life changing situation for the patient. There had been historical fears of adverse effects of surgery on mother and fetus. Despite these historic fears and risks including miscarriage, there have been consistent reports of favourable outcomes, especially in developed countries. This would influence the current and future advice given to prospective mothers in similar situations. Termination of pregnancy in these circumstances, have therefore become increasingly a nonoption for affected mothers who may want to carry on with their pregnancy as far as possible and have a healthy or fairly healthy newborn.

\section{References}

1. Danese S, Fiocci C (2011) "Ulcerative colitis". The New England Journal of Medicine 365: 1713-1725.

2. Collins S (2004) Oxford Handbook of Obstetrics and Gynaecology. vol. 2, p208.

3. Greene GG and Smith AE; Pregnancy following colectomy; prognosis; report of a case, Obstet gynecol, 1955, 5(6):793-5.

4. EBSCO host (1959) Is pregnancy safe after illeostomy and colectomy: RN. 22(5): 88-90.

5. Bertaud P, Trably F (1967) Pregnancy after total colectomy, Bull fed soc gynecol obstet. Lang Fr.

6. Koganei K, Sugita A, Shinozaki M, Kimura H, Fukushima T (1999) Pregnancy and Delivery After Total Colectomy with lleal Pouch Anal or Anal Canal Anastomosis for Ulcerative Colitis. Journal of Japan Society of Colpoproctology 52(4): 300-304.

7. Dozois EJ, Wolff BG, Tremaine WJ, Watson WJ, Drelichman ER, et al. (2006) Maternal and fetal outcome after colectomy for 
fulminant ulcerative colitis during pregnancy; case series and literature Review. Dis Colon Rectum 49(1): 64-73.

8. Greenfield C, Pounder RE, Craft IL, Lewis AA (1983) Severe Ulcerative colitis during successful pregnancy. Postgrad Med 59: 459-461.

9. Boulton R, Walker P, Pounder R (1994) Fulminant ulcerative colitis in pregnancy. Hamilton M 89(6): 931-3.

10. Wilson I, DenchJ, Garrett W (2013) Surgical management of ulcerative colitis during pregnancy, Poster presentations: Clinical: Therapy and Observation, European Crohn's and Colitis Organisation. Inflammatory Bowel Diseases.

11. Uchino M, Ikeuchi H, Matsuoka H, Bando T, Hirose K (2015) Case Rep. Gastroenterol 9(1): 74-80.

12. Fazio VW, Kiran RP, Remzi FH, Coffey JC, Heneghan HM, et al. (2013) Ileal Pouch-anal anastomosis: analysis of outcomes and quality of life in 3707 patients. Ann Surg 257: 679-685.

13. Bharadwaj S, Philpott JR, Barber MD, Graff LA, Shen B (2014) Women's health issues after ileal pouch surgery. Inflamm Bowel Dis 20:2470.

14. Juhasz ES, Fozard B, Dozois RR, Ilstrup DM, Nelson H (1995) Ileal pouch-anal anastomosis function following childbirth. An extended evaluation. Dis Colon Rectum 38:159.
15. Remzi FH, Gorgun E, Bast J, Schroeder T, Hammel J, et al. (2005)Vaginal delivery after ileal pouch-anal anastomosis: a word of caution. Dis Colon Rectum 48: 1691-1699.

16. FA. Frizelle (2016) Surgical management of ulcerative colitis.

17. Wax JR, Pinette MG, Cartin A, Blackstone J (2003) Female reproductive health after ileal pouch anal anastomosis for ulcerative colitis. Obstet Gynecol Surv 58: 270.

18. Cornish JA, Tan E, Teare J, Teoh TG, Rai R, et al. (2007) The effect of restorative proctocolectomy on sexual function, urinary function, fertility, pregnancy and delivery: a systematic review. Dis Colon Rectum 50: 1128-1138.

19. Johnson P, Richard C, Ravid A, Spencer L, Pinto E, et al. (2004) Female infertility after ileal pouch-anal anastomosis for ulcerative colitis. Dis Colon Rectum 47:1119-1126.

20. Hahnloser D, Pemberton JH, Wolff BG, Larson D, Harrington J, et al. (2004) Pregnancy and delivery before and after ileal pouchanal anastomosis for inflammatory bowel disease: immediate and long-term consequences and outcomes. Dis Colon Rectum 47: 1127-1135. 